\title{
Deconstructing major depressive episodes across unipolar and bipolar depression by severity and duration: a cross-diagnostic cluster analysis on a large, international, observational study
}

Filippo Corponi $\mathbb{C}^{1,2}$, Gerard Anmella $\mathbb{C}^{2}$, Isabella Pacchiarotti ${ }^{2,3,4}$, Ludovic Samalin², Norma Verdolini ${ }^{2,3,4}$, Dina Popovic ${ }^{5}$, Jean-Michel Azorin 6 , Jules Angst ${ }^{7}$, Charles L. Bowden ${ }^{8}$, Sergey Mosolov $\mathbb{1}^{9}$, Allan H. Young $\mathbb{B}^{10}$, Giulio Perugi ${ }^{11}$, Eduard Vieta $\mathbb{B}^{2,3,4}$ and Andrea Murru $\mathbb{B}^{2,3,4}$

\begin{abstract}
A cross-diagnostic, post-hoc analysis of the BRIDGE-II-MIX study was performed to investigate how unipolar and bipolar patients suffering from an acute major depressive episode (MDE) cluster according to severity and duration. Duration of index episode, Clinical Global Impression-Bipolar Version-Depression (CGI-BP-D) and Global Assessment of Functioning (GAF) were used as clustering variables. MANOVA and post-hoc ANOVAs examined between-group differences in clustering variables. A stepwise backward regression model explored the relationship with the 56 clinical-demographic variables available. Agglomerative hierarchical clustering with two clusters was shown as the best fit and separated the study population $(n=2314)$ into 65.73\% (Cluster $1(C 1))$ and 34.26\% (Cluster 2 (C2)). MANOVA showed a significant main effect for cluster group $(p<0.001)$ but ANOVA revealed that significant betweengroup differences were restricted to CGI-BP-D $(p<0.001)$ and GAF $(p<0.001)$, showing greater severity in C2. Psychotic features and a minimum of three DSM-5 criteria for mixed features (DSM-5-3C) had the strongest association with $C 2$, that with greater disease burden, while non-mixed depression in bipolar disorder (BD) type II had negative association. Mixed affect defined as DSM-5-3C associates with greater acute severity and overall impairment, independently of the diagnosis of bipolar or unipolar depression. In this study a pure, non-mixed depression in BD type II significantly associates with lesser burden of clinical and functional severity. The lack of association for less restrictive, researchedbased definitions of mixed features underlines DSM-5-3C specificity. If confirmed in further prospective studies, these findings would warrant major revisions of treatment algorithms for both unipolar and bipolar depression.
\end{abstract}

\section{Introduction}

The Diagnostic and Statistical Manual of Mental Disorders, Fifth Edition (DSM-5) defines a major depressive episode (MDE) as a syndromic category wherein five (or

\footnotetext{
Correspondence: Eduard Vieta (evieta@clinic.cat)

${ }^{1}$ Department of Biomedical and Neuromotor Sciences, University of Bologna, Bologna, Italy

Bipolar and Depressive Disorders Unit, Institute of Neuroscience, Hospital Clínic, University of Barcelona, IDIBAPS, CIBERSAM, Barcelona, Catalonia, Spain Full list of author information is available at the end of the article
}

more) symptoms are present and result in a clear-cut worsening of previous functioning during a minimum of 2 weeks ${ }^{1}$. DSM-5 construes MDE as unidimensional, aiming at an unachieved diagnostic reliability ${ }^{2}$, along with providing a number of specifiers defined as discrete entities. Thus, MDE is a transdiagnostic nosographic construct straddling major depressive disorder (MDD) and bipolar disorder (BD). The commonly used definitions of unipolar and bipolar depression represent an attempt to overcome this pitfall. Unipolar and bipolar depressions 
both impose a large burden for healthcare systems ${ }^{3}$. While as of today no clinical hallmark or biomarker can definitely differentiate a MDE as belonging to either MDD or $\mathrm{BD}^{4}$, depressions in MDD and BD probably do represent distinct conditions, with only partially overlapping genetic underpinnings ${ }^{5}$ and different therapeutic management ${ }^{6-8}$. Despite (hypo)mania being the earmark of $\mathrm{BD}$, depression is generally the predominant mood state, representing the most prevalent polarity at illness onset and greatest contribution to psychosocial disability ${ }^{9}$.

As opposed to the plethora of specifiers included in the DSM-5, a model mapping gradations of severity and duration might capture much of the heterogeneity in depressive burden ${ }^{10,11}$. The inclusion of specifiers in the DSM-5 tried to bridge the need to assess severity and duration whilst keeping a categorical framework ${ }^{12}$. Illness severity can be conceptualized in terms of symptoms' intensity and global functioning ${ }^{13}$. However, defining depression severity as a direct result of symptoms presence and intensity alone may be unjustified, due to the intrinsic heterogeneity of the depressive symptom as a clinical construct, and their different impact on the overall impairment that a patient may experience ${ }^{14}$. Along with the intensity of symptoms, their duration is a major element, which must be considered to perform a diagnosis of $\mathrm{MDE}^{1}$. Past studies link longer duration of index episode with greater severity ${ }^{15-19}$, higher comorbidities' burden (i.e., dysthymia and anxiety), suicidal behavior ${ }^{19}$, and lower probability of recovery, ${ }^{20}$ furthermore, duration might not be related to the index episode being a recurrent or first-onset episode ${ }^{19}$.

The BDs: Improving Diagnosis, Guidance and Education-mixed features (BRIDGE-II-MIX) study was a large, multinational, cross-sectional study, which enrolled 2811 adults aged 18 years and older with MDE according to Diagnostic and Statistical Manual of Mental Disorders, Fourth Edition Text Revision (DSM-4-TR) diagnostic criteria at the time of the consultation and applied a descriptive, bottom-up approach with the primary aim of detecting mixed symptoms among such patients ${ }^{21}$. Several subanalyses have investigated the effects of comorbidity $^{22-24}$, recurrence ${ }^{25,26}$, specific symptoms ${ }^{27-31}$, and treatment response ${ }^{32,33}$ in patients with MDE from this data set, but none looked into the impact of severity and duration.

Cluster analysis is a statistical technique that identifies subgroups in wider multidimensional or heterogeneous data, which application to multifaceted diseases, such as major depression, could help dissect disease heterogeneity, advancing diagnostic criteria, and improving treatment plans ${ }^{34-36}$.

Thus, the aim of this post-hoc study was to determine cross-diagnostic clinical clusters based on depressive burden, i.e., index episode's clinical severity, and duration, within an acutely depressed population of unipolar and bipolar patients.

\section{Method \\ Sample and assessment}

The BRIDGE-II-MIX study was a multicenter, international, non-interventional, cross-sectional study conducted between June 2009 and July 2010 and involving 239 hospital based or community psychiatrists from eight countries across three continents, i.e. Bulgaria, Egypt, Morocco, the Netherlands, Portugal, Russia, Spain, and Turkey. The study enrolled 2811 adults (aged 18 years or older), experiencing an acute MDE according to DSM-IV-TR criteria (APA, 2000). In a single consultation, psychiatrist completed a structured case report for each patients, including inclusion criteria, sociodemographic variables, psychiatric medical history and features of the depressive episode, including DSM criteria for BD, previous response to antidepressants, psychiatric comorbidity and current treatment $^{21,27}$. According to DSM-IV-TR, 735 patients (26.15\%) fulfilled criteria for BD, of whom 400 patients (14.23\%) met criteria for bipolar I disorder and 335 patients (11.92\%) for bipolar-II disorder. On the other hand, 2076 patients (73.85\%) met a DSM-IV-TR diagnosis of MDD, of whom 691 (24.58\%) were at their first MDE.

The primary objective of the BRIDGE-II-MIX study was to establish the frequency of depressive mixed states by analyzing all the relevant symptoms of either pole. After the publication of DSM-5, this was post-hoc defined as (1) the proportion of patients fulfilling DSM- 5 criteria for MDE with mixed features (American Psychiatric Association, 2013), and (2) research-based diagnostic criteria for mixed states (RBDC). DSM-5 criteria require the presence for at least a week of an MDE and at least 3 of the following (nonoverlapping) hypomanic symptoms: (1) elevated, expansive mood, (2) inflated self-esteem or grandiosity, (3) more talkative than usual or pressure to keep talking, (4) flight of ideas or subjective experience that thoughts are racing, (5) increase in energy or goaldirected activity, (6) increased or excessive involvement in activities that have a high potential for painful consequences, and (7) decreased need for sleep. The BRIDGE-II-MIX study also adopted the definition of DSM-5 subthreshold criteria for MDE with mixed features, for which the presence of an MDE plus 2 nonoverlapping hypomanic symptoms are required. RBDC are defined by the presence of MDE plus 3 out of the following 14 hypomanic symptoms for at least a week: irritable mood, affective lability, distractibility, psychomotor agitation, impulsivity, aggression (verbal or physical), racing thoughts, more talkative/pressure to keep talking, hyperactivity, increased energy, risky behavior, grandiosity, elation, and hyper-sexuality. The proportion of patients fulfilling criteria for $\mathrm{BD}$ according to the 
DSM-4-TR and bipolarity specifier proposed by Angst et al. $^{37-39}$ was also identified. The bipolarity specifier attributes a diagnosis of BD to patients who experienced an episode of elevated mood or irritable mood or increased activity with at least three of the symptoms listed under Criterion B of the DSM-4-TR, associated with at least one of the three following consequences: (1) unequivocal and observable change in functioning uncharacteristic of the person's usual behavior, (2) marked impairment in social or occupational functioning observable by others or (3) requiring hospitalization or outpatient treatment. No minimum duration was required, and no exclusion criteria were applied.

The study was conducted according to the Declaration of Helsinki (Hong Kong Amendment; http://www.wma. net) and the Good Epidemiology Practice and the International Epidemiologic Association (IEA) European Federation (http://iea.web.org). Good Epidemiologic Practice (GEP) - IEA Guidelines were followed for proper conduct of epidemiologic research, as well as pertinent national, legal, and regulatory requirements. Written informed consent was obtained from each patient. In each country, the protocol was approved by the local ethics committee.

\section{Measures of depressive severity and duration}

We took a complete cases approach, including only patients without missing observations. If missing data meets the Missing Completely at Random (MCAR) assumption, removal of observations with missing data is expected to produce unbiased estimates of means, variances, and regression weights (Allison, 2002). We used the Hawkins test of multivariate normality and heteroscedasticity, as implemented in the 'MissMech' $\mathrm{R}$ package ${ }^{40,41}$, to address this question. A nonsignificant $p$ value from this test would indicate a lack of sufficient evidence to reject the null hypothesis that data are MCAR. For this post-hoc analysis, we defined our clustering variables as: (1) duration of index episode: Collected retrospectively and measured in days. (2) Depression severity: Assessed at the time of consultation using the item for depression of the CGI-BP (CGI-BP-D $)^{42}$ which evaluates how severely ill on a scale from 1 (normal, not ill) to 7 (very severely ill) a patient is. (3) Functional impairment: estimated at the time of consultation using the Global Assessment of Functioning $(\mathrm{GAF})^{43}$, which measures how much a person's symptoms affect his or her day-to-day life on a scale of $0-100$. In line with previous recommendations, we checked that the sample size to include in the cluster analysis was no $<5 \times 2^{\mathrm{k}}(k=\text { number of clustering variables })^{42}$.

\section{Statistical analysis \\ Data normalization and exploratory analyses}

Each measure was z-transformed prior to being entered as independent variable in the cluster analysis. Clustering tendency, i.e., inherent grouping structure, was assessed using the Hopkins statistic which examines whether objects in a data set differ significantly from the assumption that they are uniformly distributed in the multidimensional space. A value close to 1 indicates highly clustered data, random data results in values around 0.5 while uniformly distributed data yield values close to $0^{44,45}$.

\section{Cluster analysis}

Optimal clustering algorithm and number of clusters were determined using the 'clValid' $\mathrm{R}$ package ${ }^{46}$, which allows for simultaneous comparison of multiple clustering algorithms and numbers of clusters in terms of validation measures. We tested for the presence of two to six clusters and we implemented three clustering methods: (1) kmeans (2) k-medoids or partitioning around medoids (PAM) and (3) agglomerative hierarchical clustering ${ }^{47,48}$. The validation measures used to compare different clustering solutions comprised internal and stability criteria. Internal criteria were calculated as connectivity, silhouette width and Dunn index. Stability criteria upon removal of each variable were: APN (average proportion of nonoverlap); $\mathrm{AD}$ (average distance); ADM (average distance between means between cluster centers); FOM (figure of merit).

Cluster group means for all measures of depressive burden, i.e., index episode duration, CGI-BP-D, and GAF, were compared using MANOVA, each measure of depressive burden was than examined separately using ANOVA to assess individual contribution. Subsequently, a stepwise backward logistic regression model was used to identify the predictive value on cluster membership of the 56 relevant clinical-demographic features available from the BRIDGE-II-MIX study. The Akaike information criterion (AIC) was used for stepwise backward variable selection. AIC performs feature selection striking a balance between goodness of fit and overfitting. Stepwise backward logistic regression was carried out using the 'MASS' R package ${ }^{49}$. Odds ratios (ORs) with $95 \%$ confidence intervals (CIs) were calculated for the observed associations.

All statistical analyses were performed using R Statistical Software (Foundation for Statistical Computing, Vienna, Austria).

\section{Results}

\section{Cluster analysis}

Out of the 2811 patients considered, after screening for missing values 2314 complete cases were included in the analysis. Missing data met the MCAR assumption, thus supporting listwise deletion as an appropriate approach. Hopkins index of 0.15 suggested inherent grouping structure. All internal validation measures and 2 out of 4 stability validation criteria favored agglomerative 
hierarchical clustering with 2 clusters as optimal clustering fit to the data set (Supplementary Fig. 1). Dendrogram produced from Ward's clustering (Supplementary Fig. 2) was cut to produce two clusters and subjects were assigned cluster membership accordingly.

$\mathrm{C} 1$ and $\mathrm{C} 2$ comprised $65.73 \%(n=1521)$ and $34.26 \%$ $(n=793)$ of the study population respectively. Using Pillai's trace, MANOVA showed a significant main effect for cluster group $\left(V=0.35, F_{3,2312}=432, p<0.001\right)$ but, as revealed by ANOVA, significant between-group differences were restricted to CGI-BP-D $\left(F_{1,2312}=190\right.$, $p<0.001)$ and $\operatorname{GAF}\left(F_{1,2312}=1297, p<0.001\right)$, both indicating greater disease severity in C2. Duration of index episode did not significantly separate the two clusters $\left(F_{1,2312}=0.34, p=0.56\right)$.

\section{Stepwise AIC backward logistic regression}

Starting with the full model including all 56 clinicaldemographic independent variables (Table 1), stepwise backward selection of independent variables reached AIC optimization at 19 variables (Table 2): 34 variables were thus removed from the full model. The final 19 variables model showed significantly better fit than the null model $\left(x^{2}=148, \mathrm{df}=19, p<0.001\right)$. In the final model, 7 out of 19 of the variables retained were significantly associated with cluster membership. Relative ORs with $95 \%$ CIs are shown in Fig. 1. Psychotic features $(\mathrm{OR}=2.12,95 \% \mathrm{CI}=$ $1.54 ; 2.93)$ was the variable most strongly associated with C2 membership, followed by a minimum of three DSM- 5 criteria for mixed features $(\mathrm{OR}=1.69,95 \% \mathrm{CI}=1.13$; $2.54)$ and history of antidepressant resistance $(\mathrm{OR}=1.63$, $95 \% \mathrm{CI}=1.33 ; 2.00)$. Years of illness $(\mathrm{OR}=1.02$, $95 \%$ $\mathrm{CI}=1.01-1.02)$ and onset age of depressive symptoms $(\mathrm{OR}=1.02,95 \% \mathrm{CI}=1.01-1.02)$ had only a tiny positive association. On the contrary, BD type II was negatively associated with $\mathrm{C} 2$ membership $(\mathrm{OR}=0.69,95 \% \mathrm{CI}=$ $0.49 ; 0.97)$.

\section{Discussion}

This post-hoc analysis of the BRIDGE-II-MIX study aimed at clustering a sample of acutely depressed patients according to measures of depressive burden, i.e., symptom intensity, functional impairment, and duration. Our analysis identified two clusters, showing little $(\mathrm{C} 1)$ and great (C2) severity. The great severity cluster $\mathrm{C} 2$ included $\sim 1$ out of 3 patients displaying a significantly greater depressive burden as defined by CGI-BP-D and GAF, whereas the duration of the index episode did not significantly contribute to clusters' separation. Among other possible explanations, such as differences in latency to access to mental health-related services, the crosssectional design of the study may account for the lack of clusters' separation by longer illness duration, since records for index episode's duration did not cover the
Table 1 The 56 clinical-demographic variables available from the BRIDGE-II-MIX study and explored in the stepwise backward logistic regression model.

\begin{tabular}{|c|c|c|c|c|}
\hline & \multicolumn{2}{|c|}{$\begin{array}{l}\text { Cluster } 1 \\
(n=1521)\end{array}$} & \multicolumn{2}{|c|}{$\begin{array}{l}\text { Cluster } 2 \\
(n=793)\end{array}$} \\
\hline & $n$ & $\%$ & $n$ & $\%$ \\
\hline \multicolumn{5}{|l|}{ Categorical variables (YES) } \\
\hline Female gender & 1057 & 69.49 & 536 & 67.59 \\
\hline Inpatient status & 366 & 24.06 & 244 & 30.76 \\
\hline $\begin{array}{l}\text { Family history of bipolar disorder } \\
\text { among first degree relatives }\end{array}$ & 230 & 15.12 & 120 & 15.13 \\
\hline \multicolumn{5}{|l|}{ Diagnosis } \\
\hline Bipolar disorder type I & 200 & 13.21 & 142 & 17.9 \\
\hline Bipolar disorder type II & 190 & 12.42 & 95 & 11.97 \\
\hline First major depressive episode & 350 & 23.01 & 168 & 21.12 \\
\hline Major depressive disorder & 781 & 51.34 & 388 & 48.92 \\
\hline
\end{tabular}

Concurrent symptoms experienced most of the time during the past week

$\begin{array}{lrrrr}\text { Anxiety } & 1211 & 79.61 & 623 & 78.56 \\ \text { Hyperphagia } & 218 & 14.33 & 114 & 14.37 \\ \text { Hypersomnia } & 244 & 16.04 & 120 & 15.13 \\ \text { Hypo-sexuality } & 935 & 61.47 & 533 & 67.21 \\ \text { Insomnia } & 1084 & 71.26 & 580 & 73.13 \\ \text { Leaden paralysis } & 364 & 23.93 & 222 & 27.99 \\ \text { Memory problems } & 935 & 60.81 & 486 & 61.28 \\ \text { Mood reactivity } & 646 & 42.47 & 317 & 39.97 \\ \text { Panic attacks } & 225 & 14.79 & 133 & 16.77 \\ \text { Psychotic features } & 85 & 5.58 & 99 & 12.48 \\ \text { Reduced appetite } & 905 & 59.50 & 514 & 64.82\end{array}$

Research-Based Diagnostic Criteria (RBDC) for mixed states

$\begin{array}{lrrrr}\text { Distractibility } & 371 & 24.39 & 214 & 26.98 \\ \text { Elation } & 65 & 4.27 & 47 & 5.92 \\ \text { Grandiosity } & 46 & 3.02 & 49 & 6.17 \\ \text { Hyperactivity } & 110 & 7.23 & 82 & 10.34 \\ \text { Hyper-sexuality } & 36 & 2.36 & 27 & 3.40 \\ \text { Impulsivity } & 214 & 14.06 & 118 & 14.88 \\ \text { Increased energy } & 90 & 5.91 & 74 & 9.31 \\ \text { Irritable mood } & 489 & 32.14 & 259 & 32.66 \\ \text { Mood lability } & 464 & 30.50 & 239 & 30.14 \\ \text { More talkative/Pressure to talk } & 168 & 11.04 & 106 & 13.36 \\ \text { Psychomotor agitation } & 224 & 14.72 & 141 & 17.78 \\ \text { Racing thoughts } & 160 & 10.51 & 110 & 13.87\end{array}$


Table 1 continued

\begin{tabular}{|c|c|c|c|c|}
\hline & \multicolumn{2}{|c|}{$\begin{array}{l}\text { Cluster } 1 \\
(n=1521)\end{array}$} & \multicolumn{2}{|c|}{$\begin{array}{l}\text { Cluster } 2 \\
(n=793)\end{array}$} \\
\hline & $n$ & $\%$ & $n$ & $\%$ \\
\hline Risky behavior & 94 & 6.18 & 71 & 8.95 \\
\hline Verbal or physical aggressivity & 194 & 12.75 & 122 & 15.38 \\
\hline \multicolumn{5}{|l|}{$\begin{array}{l}\text { Past response to antidepressant } \\
\text { treatment }\end{array}$} \\
\hline $\begin{array}{l}\text { History of (hypo)manic switches } \\
\text { ensuing antidepressant treatment }\end{array}$ & 260 & 17.09 & 163 & 20.55 \\
\hline $\begin{array}{l}\text { History of resistance to } \\
\text { antidepressant treatment }\end{array}$ & 359 & 23.60 & 278 & 35.05 \\
\hline $\begin{array}{l}\text { History of mood lability ensuing } \\
\text { antidepressant treatment }\end{array}$ & 440 & 28.92 & 269 & 33.92 \\
\hline $\begin{array}{l}\text { History of irritability ensuing } \\
\text { antidepressant treatment }\end{array}$ & 374 & 24.59 & 239 & 30.13 \\
\hline \multicolumn{5}{|l|}{ Mixed affect definitions } \\
\hline $\begin{array}{l}\text { A minimum of } 2 \text { DSM- } 5 \text { criteria for } \\
\text { mixed features }\end{array}$ & 217 & 14.26 & 147 & 18.53 \\
\hline $\begin{array}{l}\text { A minimum of } 3 \text { DSM-5 criteria for } \\
\text { mixed features }\end{array}$ & 98 & 6.44 & 93 & 11.73 \\
\hline $\begin{array}{l}\text { A minimum of } 2 \text { intradepression } \\
\text { (hypo)manic symptoms }\end{array}$ & 588 & 38.65 & 326 & 41.1 \\
\hline $\begin{array}{l}\text { A minimum of } 3 \text { intradepression } \\
\text { (hypo)manic symptoms }\end{array}$ & 420 & 27.61 & 247 & 31.14 \\
\hline \multicolumn{5}{|l|}{ Comorbidities } \\
\hline Alcohol abuse & 97 & 6.37 & 53 & 6.68 \\
\hline Borderline personality disorder & 90 & 5.91 & 67 & 8.44 \\
\hline Generalized anxiety disorder & 264 & 17.35 & 141 & 17.78 \\
\hline Obsessive-compulsive disorder & 73 & 4.79 & 43 & 5.42 \\
\hline Panic disorder & 148 & 9.73 & 89 & 11.22 \\
\hline Social phobia & 106 & 6.96 & 70 & 8.82 \\
\hline Substance abuse & 39 & 2.56 & 26 & 3.27 \\
\hline
\end{tabular}

Numeric variables

Days, if any, with (hypo)mania in the last year

Days with depression in the last year

Number of mood (depressive and/or (hypo)manic) episodes in the last year

Number of previous depressive episodes

Number of previous hospitalizations

Number of previous suicide attempts
Table 1 continued

\begin{tabular}{|c|c|c|}
\hline & $\begin{array}{l}\text { Cluster } 1 \\
(n=1521)\end{array}$ & $\begin{array}{l}\text { Cluster } 2 \\
(n=793)\end{array}$ \\
\hline & $\%$ & $\%$ \\
\hline Onset age of depressive symptoms & 34.912 .5 & 36.112 .7 \\
\hline $\begin{array}{l}\text { Total number of Research-Based } \\
\text { Diagnostic Criteria (RBDC) for } \\
\text { mixed states }\end{array}$ & 1.792 .57 & 2.092 .99 \\
\hline Years of illness & 7.799 .38 & 9.4410 .1 \\
\hline
\end{tabular}

Values across Cluster 1 and Cluster 2 are shown.

Table 2 The 19 variables selected in the final logistic regression model.

\begin{tabular}{lcccc}
\hline Variable & Coefficient & SE & $\boldsymbol{z}$ value & $\boldsymbol{p}$ value \\
\hline A minimum of 3 DSM-5 criteria & 0.53 & 0.21 & 2.54 & $0.01^{*}$ \\
for mixed features & & & & \\
Bipolar disorder type II & -0.36 & 0.17 & -2.08 & $0.04^{*}$ \\
First major depressive episode & 0.04 & 0.17 & 0.25 & 0.80 \\
Grandiosity & 0.40 & 0.26 & 1.54 & 0.12 \\
History of resistance to & 0.49 & 0.10 & 4.69 & $<0.001^{*}$ \\
antidepressant treatment & & & & \\
Hypo-sexuality & 0.19 & 0.10 & 1.90 & 0.06 \\
Impulsivity & -0.28 & 0.16 & -1.72 & 0.09 \\
Major depressive disorder & -0.23 & 0.14 & -1.72 & 0.09 \\
Memory problems & -0.18 & 0.10 & -1.84 & 0.07 \\
Mood lability & -0.23 & 0.12 & -1.93 & 0.05 \\
Mood reactivity & -0.15 & 0.10 & -1.54 & 0.12 \\
Number of mood (depressive & 0.02 & 0.01 & 1.62 & 0.10 \\
and/or (hypo)manic) episodes in & & & & \\
the last year & & & & \\
Number of previous suicide & 0.16 & 0.04 & 3.57 & $<0.001^{*}$ \\
attempts & & & & \\
Onset age of depressive & 0.02 & 0.00 & 4.02 & $<0.001^{*}$ \\
symptoms & & & & \\
Psychotic features & 0.75 & 0.16 & 4.60 & $<0.001^{*}$ \\
Racing thoughts & 0.23 & 0.16 & 1.43 & 0.15 \\
Reduced appetite & 0.19 & 0.10 & 1.93 & 0.05 \\
Verbal or physical aggressivity & 0.26 & 0.15 & 1.72 & 0.09 \\
Years of illness from onset & 0.02 & 0.01 & 3.42 & $<0.001^{*}$ \\
\hline & & & & \\
\hline
\end{tabular}

The Akaike information criterion (AIC) was used for stepwise backward variable selection, starting from the full model with 56 variables. Cluster membership (the dependent variable) was coded as 1 for Cluster 2 (positive case) and 0 for Cluster 1 (negative case).

${ }^{*} p$ values $<0.05$ 
entire course of the episode. Interestingly, the cluster including the most severe patients has BD less represented (proportionally) as diagnosis subgroup in comparison to the other cluster.

The regression model revealed that psychotic features had the largest OR for positive association with $\mathrm{C} 2$ membership (Fig. 1). The link between psychosis and depression severity is indeed long-established ${ }^{50,51}$, since the presence of psychotic features underpins cognitive and processing impairments across multiple severe mental disorders, irrespective of the clinical diagnosis ${ }^{52}$.

A key finding of our study is that the clinical presentation with mixed features according to DSM-5 criteria represents the second strongest association with the C2. Strengthening this result, pure, non-mixed presentation in acute bipolar-II depression represents the unique significant negative association with illness severity. Thus, the absence of mixed features seems a protective factor for illness severity and interestingly this was not influenced by possible treatment differences between BD types I and II. To the best of our knowledge, this finding has no precedent in scientific literature. Despite the limitations further disclosed, should it be confirmed by future, prospective evidence, this finding could have an impact on the methodology of studies designs requested by regulatory agencies. In fact, these results go against the common depiction of a severity progression spanning from unipolar depression, to BD-II and BD-I.

Overall, mixed features might be considered as a marker of severity both in research and clinical settings, outlining a functionally compromised group of patients irrespectively of the diagnosis ${ }^{53-55}$. Yet, according to our results mixed states do not invariantly represent a marker of clinical severity. In fact, the statistical contribution to $\mathrm{C} 2$ in our study was observed for mixed affect defined as the presence of at least three DSM-5 symptoms, but not as determined according to other less conservative definitions, i.e., RBDC criteria for mixed features and a minimum of two DSM-5 symptoms. This could be explained by the fact that RBDC criteria have a higher sensitivity for detection of mixed states ${ }^{56}$, but this does not directly translate into a more severe MDE, bearing doubtful acute prognostic implications. On the other hand, DSM-5 criteria, specifically a minimum of three symptoms ${ }^{1}$, seem to have higher specificity in diagnosing more severe mixed presentations, reflected by the statistical assignment to our C2.

Last, the $\mathrm{C} 2$ association in the regression model with previous suicide attempts and previous resistance to antidepressant treatments could be considered the hallmark of mixed presentation, as previously outlined in this very sample ${ }^{33}$ and previous studies on suicidal ideation and psychotic features marking more severely impaired MDE patients ${ }^{57}$. In particular, a history of previous resistance to antidepressant treatments bears important treatment implications. In fact, as suggested elsewhere, mixed-features depressions not responding to antidepressants might benefit from coupling a moodstabilizing agent or even dropping the antidepressant altogether ${ }^{58}$.

Results coming from this post-hoc analysis should be balanced against some limitations. First, due to the crosssectional design of the BRIDGE-II-MIX recall bias probably affected some of the variables' definitions, and especially our definition of duration, whose esteem might also depend on external factors (e.g., latency of referral and psychiatric assessment). Also, the assessment of symptom severity and functioning may have been somewhat hampered by the use of the GAF as a functional outcome, as its anchor points rely also on symptoms severity. This might have contributed to the strong correlation between CGI and GAF scores in our sample. Also, concerns about inter-rater reliability have been raised in the past, but its validity and clinical usefulness on the assessment of symptom and function dimensions have been established ${ }^{59}$. A deeper assessment of the overall functioning of the patients should have accounted for different dimensions that would have allowed for an increased discriminant capacity ${ }^{60}$. Second, the participating centers were not randomly selected, which may have led to a bias through the inclusion of psychiatrists with a particular interest in mixed states. However, this could also represent a study strength as some expertise is needed to detect mixed states in MDE patients. Another limitation was the wide variation in the rates of hospitalized patients across countries, ranging from 1.0 to $57.8 \%$, which could reflect economically driven policies on the use of hospitalization-based treatment rather than real differences in clinical practice or patients' severity. The main strengths of the BRIDGE-II-Mix study include the large sample size and the wide range of care settings, both hospital and community, from eight countries across three continents. Furthermore, narrow exclusion criteria increase the generalizability of the findings. Lastly, it should be acknowledged that cluster analysis is a data mining technique for finding patterns in data and is exploratory in nature.

In conclusion, in this post-hoc cluster analysis on a large international sample of 2314 acutely depressed patients, approximately one third of the study population fitted into a significantly more severe cluster of patients, independently of the traditional, categorical diagnosis. Mixed states seem to challenge the traditional unipolar-bipolar dichotomy and bridge the gap between these two categories of mood disorders. Besides well-documented and straightforward associations, such as current psychotic symptoms, history of resistance to antidepressant treatment and a history of suicide attempts, mixed affect 


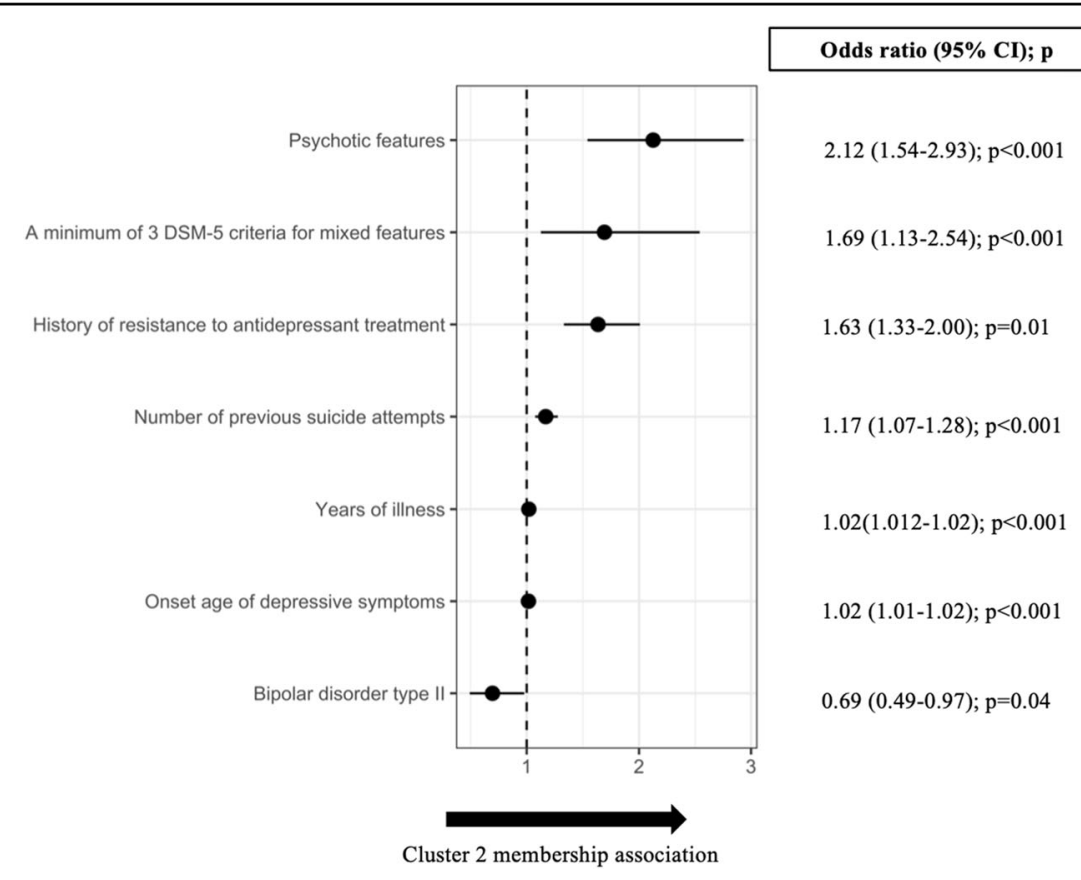

Fig. 1 Odds ratios (ORs) with $95 \%$ confidence intervals (95\% Cls) are shown for the 7 variables with $p$ value $<0.05$ out of the 19 variables in the final stepwise Akaike information criterion (AIC) backward logistic regression model. ORs are expressed with respect to Cluster 2 , i.e., the cluster displaying grater depressive burden.

defined as the co-occurrence of a minimum of three DSM-5 criteria for mixed features was significantly associated to more severe acute depression, supporting the usefulness of this definition for mixed features as significantly relevant to depression severity.

According to our results, a BD diagnosis does not automatically imply a worse severity of depression. In contrast, mixed features according to the current nosology should be considered as an intrinsic marker of severity, independently of a bipolar/unipolar diagnosis, in acutely depressed patients. This points to a continuum of the mood spectrum as a unitary phenomenon.

\section{Acknowledgements}

The authors thank the support of the Spanish Ministry of Science, Innovation (PI15/00283-PI18/00805) and cofinanced by the Instituto Carlos III integrated into the Plan Nacional de I+D+I (cofounded by European Regional Development Fund/European Social Fund) 'investing in your future"; the CIBERSAM (Centro de Investigación Biomédica en Red de Salud Mental); the Secretaria d'Universitats i Recerca del Departament d'Economia i Coneixement (2017 SGR 1365) and the CERCA Programme/Generalitat de Catalunya. We thank all the authors of the included papers and study participants. Prof Young's research is funded by the National Institute for Health Research (NIHR) Biomedical Research Centre at South London and Maudsley NHS Foundation Trust and King's College London. The views expressed are those of the authors and not necessarily those of the NHS, the NIHR, or the Department of Health.

\section{Funding}

This study is sponsored by Sanofi-Aventis. All investigators recruited received fees from the sponsor in recognition of their participation in the study on a per-patient basis. The present study received no funding.

\section{Author details}

'Department of Biomedical and Neuromotor Sciences, University of Bologna, Bologna, Italy. ${ }^{2}$ Bipolar and Depressive Disorders Unit, Institute of Neuroscience, Hospital Clínic, University of Barcelona, IDIBAPS, CIBERSAM, Barcelona, Catalonia, Spain. ${ }^{3}$ Biomedical Research Networking Center for Mental Health (CIBERSAM), Barcelona, Spain. ${ }^{4}$ August Pi I Sunyer Biomedical Research Institute (IDIBAPS), Barcelona, Spain. ${ }^{5}$ Psychiatry B, Chaim Sheba Medical Center, Ramat-Gan, Israel. ${ }^{6}$ Department of Psychiatry, Sainte Marguerite Hospital, Marseille, France. 'Department of Psychiatry, University of Zurich, Zurich, Switzerland. ${ }^{8}$ Department of Psychiatry, University of Texas Health Science Center, San Antonio, TX, USA. ${ }^{9}$ Department for Therapy of Mental Disorders, Moscow Research Institute of Psychiatry, Moscow, Russia.

${ }^{10}$ Department of Psychological Medicine, Institute of Psychiatry, Psychology \& Neuroscience, King's College London, Centre for Affective Disorders, London, UK. ${ }^{11}$ Clinica Psichiatrica, University of Pisa, Pisa, Italy

\section{Code availability}

All R-code we developed for statistical modeling is available upon request.

\section{Conflict of interest}

J.A., F.C., G.A., and N.V. declare no conflict of interest. I.P. has received CMErelated honoraria, or consulting fees from ADAMED, Janssen-Cilag, and Lundbeck. L.S. has received grants, honoraria, or consulting fees from JanssenCilag, Lundbeck, and Otsuka. D.P. has served as a speaker and/or medical writer for Bristol-Myers Squibb, Merck Sharp \& Dohme, Janssen-Cilag, and Ferrer. J.M. A. has received research support and has acted as a consultant and/or served on a speaker's bureau for Janssen, Lundbeck, Otsuka, Roche, Servier, and Takeda, and reports no financial or other relationship relevant to the subject of this article. C.L.B. has received grant support from Sunovion and the NIMH, and has consulted for Takeda, and reports no financial or other relationship relevant to the subject of this article. S.M. has received research grants from, and been involved in clinical trials for Servier, Eli Lilly, Lundbeck, AstraZeneca, Janssen-Cilag, Sanofi-Aventis, and Geodon Richter and reports no financial or other relationship relevant to the subject of this article. A.H.Y. is honorary Consultant SLaM (NHS, UK). He provided lectures and advisory boards for the following companies with drugs used in affective and related disorders: Astrazenaca, Eli Lilly, Janssen, Lunbeck, Sunovion, Servier, Livanova, and 
Janssen. He does not share holdings in pharmaceutical companies. He is Lead Investigator for Embolden Study (AZ), BCI Neuroplasticity study and Aripiprazole Mania Study, he did investigator initiated studies from AZ, Eli Lilly, Lundbeck, Wyeth. Grant funding (past and present): NIMH (USA); CIHR (Canada); NARSAD (USA); Stanley Medical Research Institute (USA); MRC (UK); Wellcome Trust (UK); Royal College of Physicians (Edin); BMA (UK);UBC-VGH Foundation (Canada); WEDC (Canada); CCS Depression Research Fund (Canada); MSFHR (Canada); NIHR (UK). Janssen (UK). He reports no financial or other relationship relevant to the subject of this article. G.P. has acted as consultant of Lundbeck, Angelini, FBHealth. He received grant/research support from Lundbeck and Angelini. He is on the speaker/advisory board of Sanofi-Aventis, Lundbeck, FBHealth, Angelini, and reports no financial or other relationship relevant to the subject of this article. E.V. has received grants and served as consultant, advisor or CME speaker for the following entities: $A B-$ Biotics,Abbott, Allergan, Angelini, AstraZeneca, Bristol-Myers Squibb, Dainippon Sumitomo Pharma, Farmindustria, Ferrer, Forest Research Institute, Gedeon Richter, Glaxo-Smith-Kline, Janssen, Lundbeck, Otsuka, Pfizer, Roche, SAGE, Sanofi-Aventis, Servier, Shire, Sunovion, Takeda, the Brain and Behaviour Foundation, the Spanish Ministry of Science and Innovation (CIBERSAM), the EU Horizon 2020, and the Stanley Medical Research Institute. A.M. has received grants, honoraria, or consulting fees from, Janssen, Lundbeck, and Otsuka.

\section{Publisher's note}

Springer Nature remains neutral with regard to jurisdictional claims in published maps and institutional affiliations.

Supplementary Information accompanies this paper at (https://doi.org/ 10.1038/s41398-020-00922-2).

Received: 20 February 2020 Revised: 7 June 2020 Accepted: 9 June 2020 Published online: 19 July 2020

\section{References}

1. American Psychiatric Association. Diagnostic and Statistical Manual of Mental Disorders (American Psychiatric Association, Washington, DC, 2013).

2. Freedman, R. et al. The initial field trials of DSM-5: new blooms and old thorns. Am. J. Psychiatry 170, 1-5 (2013).

3. WHO. Depression: Fact Sheet (World Health Organisation, Geneva, 2017). http:// www.who.int/mediacentre/factsheets/fs369/en/.

4. Hirschfeld, R. M. Differential diagnosis of bipolar disorder and major depressive disorder. J. Affect Disord. 169(Suppl 1), S12-S16 (2014).

5. Milaneschi, Y. et al. Polygenic dissection of major depression clinical heterogeneity. Mol. Psychiatry 21, 516-522 (2016).

6. Yatham, L. N. et al. Canadian Network for Mood and Anxiety Treatments (CANMAT) and International Society for Bipolar Disorders (ISBD) 2018 guidelines for the management of patients with bipolar disorder. Bipolar Disord. 20, 97-170 (2018).

7. Grunze, H. et al. The World Federation of Societies of Biological Psychiatry (WFSBP) Guidelines for the Biological Treatment of Bipolar Disorders: update 2010 on the treatment of acute bipolar depression. World J. Biol. Psychiatry 11, 81-109 (2010).

8. Grunze, $H$. et al. The World Federation of Societies of Biological Psychiatry (WFSBP) Guidelines for the Biological Treatment of Bipolar Disorders: acute and long-term treatment of mixed states in bipolar disorder. World J. Biol. Psychiatry 19, 2-58 (2018).

9. Bonnin, C. M. et al. Clinical and neurocognitive predictors of functional outcome in bipolar euthymic patients: a long-term, follow-up study. J. Affect Disord. 121, 156-160 (2010).

10. Klein, D. N. Classification of depressive disorders in the DSM-V: proposal for a two-dimension system. J. Abnorm. Psychol. 117, 552-560 (2008).

11. Blom, M. B. et al. Severity and duration of depression, not personality factors, predict short term outcome in the treatment of major depression. J. Affect Disord. 104, 119-126 (2007).

12. Zimmerman, M., Balling, C., Chelminski, I. \& Dalrymple, K. Understanding the severity of depression: which symptoms of depression are the best indicators of depression severity? Compr. Psychiatry 87, 84-88 (2018).
13. Zimmerman, M., Morgan, T. A. \& Stanton, K. The severity of psychiatric disorders. World Psychiatry 17, 258-275 (2018).

14. Fried, E. I. \& Nesse, R. M. Depression is not a consistent syndrome: an investigation of unique symptom patterns in the STAR*D study. J. Affect Disord. 172 96-102 (2015).

15. Spijker, J. et al. Duration of major depressive episodes in the general population: results from The Netherlands Mental Health Survey and Incidence Study (NEMESIS). Br. J. Psychiatry 181, 208-213 (2002).

16. Keller, M. B., Shapiro, R. W., Lavori, P. W. \& Wolfe, N. Recovery in major depressive disorder: analysis with the life table and regression models. Arch Gen. Psychiatry 39, 905-910 (1982).

17. Mueller, T. I. et al. Recovery after 5 years of unremitting major depressive disorder. Arch. Gen. Psychiatry 53, 794-799 (1996).

18. Furukawa, T. A., Kitamura, T. \& Takahashi, K. Time to recovery of an inception cohort with hitherto untreated unipolar major depressive episodes. Br. J. Psychiatry 177, 331-335 (2000).

19. Ten Have, M. et al. Duration of major and minor depressive episodes and associated risk indicators in a psychiatric epidemiological cohort study of the general population. Acta Psychiatr. Scand. 136, 300-312 (2017).

20. Keller, M. B. et al. Time to recovery, chronicity, and levels of psychopathology in major depression. A 5-year prospective follow-up of 431 subjects. Arch. Gen Psychiatry 49, 809-816 (1992).

21. Perugi, G. et al. Mixed features in patients with a major depressive episode: the BRIDGE-II-MIX study. J. Clin. Psychiatry 76, e351-e358 (2015).

22. Perugi, G. et al. Relationships between mixed features and borderline personality disorder in 2811 patients with major depressive episode. Acta Psychiatr. Scand. 133, 133-143 (2016).

23. Petri, E. et al. Obesity in patients with major depression is related to bipolarity and mixed features: evidence from the BRIDGE-II-Mix study. Bipolar Disord. 19 458-464 (2017).

24. Vannucchi, G. et al. The relationship between attention deficit hyperactivity disorder, bipolarity and mixed features in major depressive patients: evidence from the BRIDGE-II-Mix Study. J. Affect Disord. 246, 346-354 (2019).

25. Mazzarini, L. et al. Is recurrence in major depressive disorder related to bipolarity and mixed features? Results from the BRIDGE-II-Mix study. J. Affect Disord. 229, 164-170 (2018).

26. Barbuti, M. et al. Relationships between recurrence and polarity in major depressive disorders: pooled analysis of the BRIDGE and BRIDGE-II-MIX cohorts. J. Affect Disord. 256, 250-258 (2019).

27. Popovic, D. et al. Suicide attempts in major depressive episode: evidence from the BRIDGE-II-Mix study. Bipolar Disord. 17, 795-803 (2015).

28. Verdolini, N. et al. Aggressiveness in depression: a neglected symptom possibly associated with bipolarity and mixed features. Acta Psychiatr. Scand. 136, 362-372 (2017)

29. Murru, A. et al. The implications of hypersomnia in the context of major depression: results from a large, international, observational study. Eur. Neuropsychopharmacol. 29, 471-481 (2019).

30. Brancati, G. E. et al. The role of overlapping excitatory symptoms in major depression: are they relevant for the diagnosis of mixed state? J. Psychiatr. Res. 115, 151-157 (2019).

31. Verdolini, N., et al. Sultans of swing: a reappraisal of the intertwined association between affective lability and mood reactivity in a post hoc analysis of the BRIDGE-II-MIX Study. J Clin Psychiatry 80, 17m12082 (2019).

32. Barbuti, M. et al. Antidepressant-induced hypomania/mania in patients with major depression: Evidence from the BRIDGE-II-MIX study. J. Affect Disord. 219, 187-192 (2017).

33. Perugi, G. et al. Patterns of response to antidepressants in major depressive disorder: Drug resistance or worsening of depression are associated with a bipolar diathesis. Eur. Neuropsychopharmacol. 29, 825-834 (2019).

34. Lee, J. et al. Deconstructing bipolar disorder and schizophrenia: a crossdiagnostic cluster analysis of cognitive phenotypes. J. Affect Disord. 209, 71-79 (2017).

35. Sharpley, C. F., Bitsika, V., Warren, A. K. \& Christie, D. R. H. Using cluster analysis of anxiety-depression to identify subgroups of prostate cancer patients for targeted treatment planning. Psychooncology 26, 1846-1851 (2017).

36. Alden, E. C., Cobia, D. J., Reilly, J. L. \& Smith, M. J. Cluster analysis differentiates high and low community functioning in schizophrenia: subgroups differ on working memory but not other neurocognitive domains. Schizophr. Res. 168, 273-278 (2015) 
37. Angst, J. Bipolar disorders in DSM-5: strengths, problems and perspectives. Int J. Bipolar Disord. 1, 12 (2013).

38. Angst, J. \& Cassano, G. The mood spectrum: improving the diagnosis of bipolar disorder. Bipolar Disord. 7(Suppl 4), 4-12 (2005).

39. Angst, J. et al. Prevalence and characteristics of undiagnosed bipolar disorders in patients with a major depressive episode: the BRIDGE study. Arch. Gen. Psychiatry 68, 791-798 (2011).

40. Jamshidian, M., Jalal, S. \& Jansen, C. MissMech: An R package for testing homoscedasticity, multivariate normality, and missing completely at random (MCAR). J. Stat. Softw. 56, 1-31 (2014).

41. Jamshidian, M. \& Jalal, S. Tests of homoscedasticity, normality, and missing completely at random for incomplete multivariate data. Psychometrika 75, 649-674 (2010).

42. Spearing, M. K., Post, R. M., Leverich, G. S., Brandt, D. \& Nolen, W. Modification of the Clinical Global Impressions (CGl) Scale for use in bipolar illness (BP): the CGI-BP. Psychiatry Res. 73, 159-171 (1997).

43. Endicott, J., Spitzer, R. L., Fleiss, J. L. \& Cohen, J. The global assessment scale. A procedure for measuring overall severity of psychiatric disturbance. Arch. Gen. Psychiatry 33, 766-771 (1976).

44. Hopkins, B. \& Skellam, J. G. A new method for determining the type of distribution of plant individuals. Ann. Bot. 18, 213-227 (1954).

45. Banerjee, A. Validating clusters using the Hopkins statistic. IEEE International Conference on Fuzzy Systems, 149-153 (2004).

46. Brock, G., Datta, S. \& Pihur, V. clValid: an R package for cluster validation. J. Stat Softw. 25, 1-22 (2008).

47. McLachlan, G. J. Cluster analysis and related techniques in medical research. Stat. Methods Med. Res. 1, 27-48 (1992).

48. Frades, I. \& Matthiesen, R. Overview on techniques in cluster analysis. Methods Mol. Biol. 593, 81-107 (2010).

49. Ripley, B., Bates, D., Hornik, K., Gebhardt, A. \& Firth, D. Package 'MASS'. cran.rproject.org/web/packages/MASS (2011).
50. Buoli, M., Caldiroli, A. \& Altamura, A. C. Psychotic versus non-psychotic major depressive disorder: a comparative naturalistic study. Asian J. Psychiatr. 6, 333-337 (2013).

51. Dold, M., et al. Psychotic features in patients with major depressive disorder: a report from the european group for the study of resistant depression. J Clin Psychiatry 80,17m12090 (2019).

52. Baker, J. T. et al. Functional connectomics of affective and psychotic pathology. Proc. Natl Acad. Sci. USA 116, 9050-9059 (2019).

53. Shim, I. H., Woo, Y. S., Jun, T. Y. \& Bahk, W. M. Mixed-state bipolar I and ॥ depression: time to remission and clinical characteristics. J. Affect Disord. 152154, 340-346 (2014).

54. Malhi, G. S. et al. Mixed state discrimination: a DSM problem that wont go away? J. Affect Disord. 158, 8-10 (2014).

55. Verdolini, N. et al. Mixed states in bipolar and major depressive disorders: systematic review and quality appraisal of guidelines. Acta Psychiatr. Scand. 138, 196-222 (2018).

56. Pacchiarotti, I. et al. Mixed Features in Depression: the Unmet Needs of Diagnostic and Statistical Manual of Mental Disorders. Fifth Edition (Psychiatric Clinics) (2020).

57. Cassano, G. B. et al. The mood spectrum in unipolar and bipolar disorder: arguments for a unitary approach. Am. J. Psychiatry 161, 1264-1269 (2004).

58. Stahl, S. M. et al. Guidelines for the recognition and management of mixed depression. CNS Spectr. 22, 203-219 (2017).

59. Pedersen, G. \& Karterud, S. The symptom and function dimensions of the Global Assessment of Functioning (GAF) scale. Compr. Psychiatry 53, 292-298 (2012).

60. Rosa, A. R. et al. Validity and reliability of the Functioning Assessment Short Test (FAST) in bipolar disorder. Clin. Pract. Epidemiol. Ment. Health 3, 5 (2007). 